\title{
Exercício físico na Dibetes Mellitus, uma revisão narrativa
} Physical exercise in Diabetes Mellitus, a narrative review

Karine Zenatti Ely, ${ }^{1}$ Flávio da Silva Spode, ${ }^{1}$ Raquel Cristine Barcella, Isabel Viegas Schonhofen, ${ }^{1}$ Dulciane Paiva,' Hildegard Hedwig Pohl,' Lia Gonçalves Possuelo' 'Universidade de Santa Cruz do Sul (Unisc), Santa Cruz do Sul, RS, Brasil.

Recebido em: 01/10/2017 / Aceito em: 21/12/2017 / Publicado em: 30/12/2017

karine-ely@saude.rs.gov.br

\section{RESUMO}

O Diabetes Melllitus caracteriza-se pela hiperglicemia. Os exercícios físicos apresentam efeitos hipoglicemiantes, sendo importantes coadjuvantes no tratamento do Diabetes Mellitus Tipo 1 (DM1) e do Diabetes Mellitus Tipo 2 (DM2). Objetivo: identificar o tipo de exercício físico, volume e intensidade ideal para pacientes com Diabetes Mellitus, considerando as disfunções metabólicas e as variáveis cardiorrespiratórias. Método: trata-se de uma revisão narrativa que utilizou como fonte de buscas as bases de dados LILACS, Scielo e Medline (PubMed), com os seguintes descritores: Diabetes Mellitus, metabolismo dos carboidratos, exercício e glicemia. Resultados: foram selecionados 10 artigos para análise e discussão que avaliaram variáveis cardiorrespiratórias nas disfunções metabólicas durante o exercício físico do DM1 e DM2. Considerações finais: não foi possível concluir qual o tipo de exercício físico, volume e intensidade é ideal para pacientes com DM1 e DM2. Para aproveitar ao máximo todos os benefícios dos exercícios físicos é relevante que os mesmos sejam estruturados de forma individualizada, considerando a alimentação, a administração de insulina exógena conforme a duração e a intensidade da atividade física. Desta forma, será possível obter resultados positivos em relação à capacidade cardiorrespiratória e a regulação da glicose e da insulina.

Palavras-chave: Diabetes Mellitus; Metabolismo dos carboidratos; Exercício; Glicemia.

\section{ABSTRACT}

Diabetes Mellitus is characterized by hyperglycemia. Physical exercises have hypoglycemic effects and are important adjuncts in the treatment of Type 1 Diabetes Mellitus (DM1) and Type 2 Diabetes Mellitus (DM2). Objective: to identify the type of physical exercise, volume and intensity ideal for patients with Diabetes Mellitus, considering the metabolic dysfunctions and the cardiorespiratory variables. Method: this is a narrative review that used the LILACS, Scielo and Medline (PubMed) databases as the source of searches, with the following descriptors: Diabetes mellitus, carbohydrate metabolism, exercise and blood glucose. Results: 10 articles were selected for analysis and discussion that evaluated cardiorespiratory variables in metabolic dysfunctions during physical exercise of DM1 and DM2. Closing remarks: it was not possible to conclude the type of physical exercise, volume and intensity is ideal for patients with DM1 and DM2. To take full advantage of all the benefits of physical exercise, it is important that they are structured in an individualized way, considering the diet, the administration of exogenous insulin according to the duration and intensity of physical activity. In this way, it will be possible to obtain positive results in relation to the cardiorespiratory capacity and the regulation of glucose and insulin.

Keywords: Diabetes Mellitus; Carboydrate metabolism; Exercise; Blood glucose. 


\section{INTRODUÇÃO}

O Diabetes Mellitus (DM) é um “grupo heterogêneo de distúrbios metabólicos que apresenta em comum a hiperglicemia". ${ }^{1}$ Em 2015, representou mais de 12\% dos gastos totais com saúde no mundo, tem sido responsável por 1,5 milhões de óbitos, subindo para sexta posição no ranking das patologias que mais matam. ${ }^{2} \mathrm{~A}$ Federação Internacional de Diabetes (IDF) estima 387 milhões de doentes no mundo, que poderão chegar a 471 milhões de pessoas até 2035. Devido a sua magnitude, tem sido tratada como uma epidemia em curso. ${ }^{3}$

No Brasil, estima-se prevalência do DM pode atingir até $20 \%$ da população, na faixa etária de 35 a 74 anos, significando 11,9 milhões de pessoas doentes e podendo atingir 19,2 milhões até 2035. A prevalência de DM é heterogênea na população, variando de acordo com o sexo, faixa etária, grau de escolaridade, descendência étnica, entre outros fatores. A mortalidade em 2011, foi de 33,7 por 100 mil habitantes na população geral.'

O DM é originada por distúrbios no metabolismo de nutrientes como carboidratos, proteínas e gorduras, além de problemas na secreção ou ação da insulina e manifesta-se em duas formas principais: Diabetes Mellitus tipo 1 (DM1) e Diabetes Mellitus tipo II (DM2). ${ }^{4}$ O DM1 é uma doença metabólica autoimune, caracterizada pela "destruição das células beta nas ilhotas pancreáticas" e o DM2 é uma doença metabólica complexa caracterizada por "resistência à insulina, hiperglicemia e disfunção vascular". ${ }^{5}$

Diante do exposto, a presente revisão narrativa teve como objetivo identificar o tipo de exercício físico, volume e intensidade ideal para pacientes com Diabetes Mellitus, considerando as disfunções metabólicas e as variáveis cardiorrespiratórias.

\section{METODOLOGIA}

Trata-se de uma revisão narrativa de literatura sobre as alterações metabólicas decorrentes do diabetes e sua influência nas variáveis cardiorrespiratórias, por meio da técnica de investigação documental e análise de artigos científicos. Foram utilizados como fonte de pesquisa artigos científicos publicados em periódicos indexados nas bases de dados LILACS, Scielo e Medline (PubMed), com os seguintes descritores: Diabetes mellitus, metabolismo dos carboidratos, exercício e glicemia.
Os critérios de inclusão foram artigos científicos completos, publicados entre 2013 e 2017, em português e inglês, sobre Diabetes mellitus, derivados de pesquisas sobre alterações no metabolismo dos carboidratos e exercícios físicos, que consideraram variáveis cardiorrespiratórias. Foram excluídos estudos que não foram realizados com humanos, publicados em outros idiomas, realizados com gestantes e atletas, para testagem de medicamentos e que não considerassem as variáveis cardiorrespiratórias.

A pesquisa resultou em 270 artigos. Utilizando os critérios de inclusão, foram obtidos 173 artigos. Aos critérios de exclusão foram pré-selecionados 27 artigos científicos para leitura dos resumos. Alguns resumos não foram esclarecedores e prescindiram leitura completa para identificar a inclusão ou exclusão na pesquisa. Foram identificados 10 artigos que contemplavam o objetivo do estudo e estes foram selecionados para análise. Da análise, surgiram duas linhas de discussão, a primeira relaciona variáveis cardiorrespiratórias ao exercício físico na DM1 e a segunda relaciona as mesmas variáveis à DM2.

\section{RESULTADOS}

Esta revisão narrativa selecionou 10 artigos para análise e discussão que avaliaram variáveis cardiorrespiratórias nas disfunções metabólicas durante o exercício físico do DM1 e DM2. Os estudos selecionados incluíam cinco artigos referentes à DM1 e cinco artigos referentes à DM2 e foram publicados nos seguintes periódicos: Revista Brasileira de Medicina do Esporte, Diabetologia, Current Opinion in Clinical Nutrition \& Metabolic Care, Comprehensive Physiology, PLoS One, The Journal of Clinical Endocrinology \& Metabolism e Medicine and Science in Sports and Exercise.

A tabela 1 apresenta a síntese dos artigos selecionados publicados entre 2014 e 2017, sendo a maioria em 2015, totalizando quatro estudos. Quatro dos artigos utilizam-se da metodologia do caso controle, buscando identificar semelhanças e diferenças de diversos tipos de exercícios entre pessoas com DM1 e DM2 e pessoas que não apresentam estas patologias. Foram selecionados também um estudo descritivo transversal, um estudo prospectivo quase experimental, dois ensaios clínicos controlados e randomizado e dois artigos de revisão. Apenas dois artigos estão publicados em língua portuguesa e os demais em língua inglesa.

Tabela 1 - Estudos selecionados na pesquisa.

\begin{tabular}{|c|c|c|c|c|}
\hline Titúlo do Artigo & Ano de Publicação & Metodologia & Objetivos & Conclusões \\
\hline $\begin{array}{l}\text { Efeito agudo dos exercí- } \\
\text { cios intermitentes sobre a } \\
\text { glicemia de adolescentes } \\
\text { com DM1 }\end{array}$ & $\begin{array}{l}2017 \\
\text { Revista Brasileira de } \\
\text { Medicina do Esporte }\end{array}$ & $\begin{array}{l}\text { Estudo descritivo } \\
\text { transversal }\end{array}$ & $\begin{array}{l}\text { Verificar o efeito agudo dos exercí- } \\
\text { cios intermitentes sobre glicemia e } \\
\text { oxidação de substratos energéticos } \\
\text { em adolescentes com DM1. }\end{array}$ & $\begin{array}{l}\text { Espera-se melhor controle glicêmico em } \\
\text { adolescentes com DM1 para adolescentes } \\
\text { com maior score Z do IMC (Índice de Massa } \\
\text { Corporal). }\end{array}$ \\
\hline $\begin{array}{l}\text { Resposta metabólica e } \\
\text { hormonal ao exercício inter- } \\
\text { mitente de alta intensidade } \\
\text { e intensidade moderada } \\
\text { contínua em indivíduos } \\
\text { com DM1: estudo cruzado } \\
\text { aleatorizado }\end{array}$ & $\begin{array}{l}2016 \\
\text { Diabetologia }\end{array}$ & $\begin{array}{l}\text { Ensaio clínico } \\
\text { prospectivo } \\
\text { randomizado } \\
\text { e aberto }\end{array}$ & $\begin{array}{l}\text { Investigar o metabolismo do } \\
\text { combustível relacionado ao exer- } \\
\text { cício no IHE (intermitente de alta } \\
\text { intensidade) e CONT (intensidade } \\
\text { moderada contínua) em indivíduos } \\
\text { com DM1 }\end{array}$ & $\begin{array}{l}\text { Indivíduos com DM1 sem utilização de insu- } \\
\text { lina que realizaram IHE reduziram os níveis de } \\
\text { glicose exógena em comparação com CONT. }\end{array}$ \\
\hline
\end{tabular}


Metabolismo de glicose associado ao exercício em indivíduos com DM1

\section{5}

Current Opinion in

Clinical Nutrition \&

Metabolic Care.

\section{Artigo de \\ Revisão}

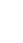

\begin{tabular}{lll}
\hline Exercício e DM1 & 2013 & Artigo de \\
& Comprehensive & revisão \\
Physiology &
\end{tabular}

\begin{tabular}{|c|c|c|}
\hline $\begin{array}{l}\text { Taxa de oxidação da glicose } \\
\text { no corpo inteiro durante o } \\
\text { exercício em pacientes com } \\
\text { DM1 em condições de vida } \\
\text { usuais }\end{array}$ & $\begin{array}{l}2013 \\
\text { Metabolism }\end{array}$ & Caso Controle \\
\hline $\begin{array}{l}\text { O treinamento intervalado } \\
\text { de alta intensidade melhora } \\
\text { o controle glicêmico e a fun- } \\
\text { ção das células } \beta \text { pancreáti- } \\
\text { cas de pacientes com DM2 }\end{array}$ & $\begin{array}{l}2015 \\
\text { PLoS One }\end{array}$ & Caso Controle \\
\hline $\begin{array}{l}\text { Efeito agudo do exercício } \\
\text { aeróbico na glicemia em } \\
\text { diabéticos } 2 \text { sob medicação }\end{array}$ & $\begin{array}{l}2015 \\
\text { Revista Brasileira de } \\
\text { Medicina do Esporte }\end{array}$ & $\begin{array}{l}\text { Prospectivo } \\
\text { quase experi- } \\
\text { mental }\end{array}$ \\
\hline $\begin{array}{l}\text { Efeitos agudos do exercício } \\
\text { intervalado versus exercício } \\
\text { de caminhada continua } \\
\text { sobre o controle glicêmico } \\
\text { em indivíduos com DM2 }\end{array}$ & $\begin{array}{l}2014 \\
\text { The Journal of Clini- } \\
\text { cal Endocrinology \& } \\
\text { Metabolism }\end{array}$ & $\begin{array}{l}\text { Crossover, } \\
\text { estudo } \\
\text { controlado }\end{array}$ \\
\hline $\begin{array}{l}\text { Exercícios intensos alteram } \\
\text { a glicemia pós-prandial de } \\
\text { vida livre na DM2 }\end{array}$ & $\begin{array}{l}2014 \\
\text { Medicine and Sci- } \\
\text { ence in Sports and } \\
\text { Exercise }\end{array}$ & Caso controle \\
\hline
\end{tabular}

\begin{tabular}{lll}
\hline DM2 altera as assinaturas & 2015 & e 48h pós exercício. \\
$\begin{array}{l}\text { metabólica e transcricional } \\
\text { do metabolism de glicose }\end{array}$ & Diabetologia & Investigar o efeito da DM2 nas mu- \\
danças metabólicas plasmáticas e a \\
resposta transcricional muscular.
\end{tabular}

Resumir o conhecimento disponível sobre o metabolismo da glicose associado ao exercício em indivíduos com DM1.
Os indivíduos com DM1 não diferem em sua resposta de glicose ao exercício quando comparados com indivíduos saudáveis se os estudos forem realizados em condições padronizadas com insulina e níveis de glicose mantidos próximos às faixas fisiológicas.

O exercício físico é benéfico para a prevenção de complicações cardiovasculares, porém alterações do tecido muscular e da memória metabólica com episódios de hipoglicemia e hiperglicemia afetam a resposta adequada à atividade física. Os benefícios superam as limitações devendo o exercício físico sempre ser estimulado.

Não houve diferença significativa de GLUox em pacientes com DM1 e o grupo controle.

Glicose (GLUox) em pacientes com DM1, comparados com indivíduos em condições normais de vida durante o exercício prolongado (3 h). Investigar o controle glicêmico, a função pancreática e a massa total de gordura antes e após 8 semanas de treinamento intervalado de alta intensidade e baixo volume (HIIT). Verificar o efeito agudo do exercício sobre a glicemia capilar em indivíduos diabéticos em uso de insulina ou antidiabéticos orais Determinar se o exercício baseado em intervalo melhora a tolerância à glicemia pós-prandial e a glicemia de vida livre mais do que o exercício contínuo.

Investigar o efeito de um exercício intenso em curto período de tempo realizado pela manhã e sua alteração metabólica na Glicemia pós-prandial (PPG) na DM2 em 24h e 48h pós exercício. glicólise e mediadores de insulina em participantes diabéticos quando comparados aos controles.

O HIIT diminuiu a massa abdominal nos dois grupos e melhora o controle glicêmico e a função das células beta-pancreáticas em pacientes com DM2.

O exercício tem ação hipoglicemiante, entretanto, nos indivíduos com alterações no me-

Uma sessão de exercício intervalado melhora mais o controle glicêmico do que a caminhada contínua, porém ambos são mais eficazes que o grupo controle.

O exercício intenso em curto período diminui a glicemia de forma significativa até a segunda refeição após intervenção, sugerindo atividades físicas diárias. tabolismo dos carboidratos não é tão evidente.

\section{DISCUSSÃo}

Os artigos selecionados apresentam dados nos quais os exercícios físicos em pessoas hígidas convergem para estilos de vida mais saudáveis e equilibrados, prevenindo, atrasando ou limitando as patologias crônicas mais prevalentes. Em pessoas com DM1 e DM2, os exercícios melhoram a resistência à insulina, controlam o peso corporal, são coadjuvantes no tratamento de problemas psicossociais, e à longo prazo, são considerados fatores de proteção para doenças cardiovasculares. ${ }^{6-10}$

\section{EXERCÍ́CIO FÍSICO NA DIABETES TIPO 1}

A destruição das células beta do pâncreas leva a necessidade de utilização de insulina exógena pelos pessoas que apresentam DM1. Alterações no metabolismo dos carboidratos resulta em hiperglicemia e danos lentos e graduais ao organismo. A utilização de insulina exógena provoca flutuações glicêmicas, alterando a qualidade de vida cotidiana e consequentemente, a realização de atividades físicas. A resposta aguda ao exercício no diabético pode ocorrer de forma inadequa- da, não correspondendo a necessidade de aumento de energia necessária para realização do exercício. ${ }^{6,11}$

Indivíduos com DM1 apresentam redução do tamanho da fibra muscular e mudanças na distribuição das mesmas. A atrofia muscular é relativa e estudos sugerem que a insulina diminui a degradação proteica do músculo, mas não atua no aumento da síntese proteica. A redução da força muscular, também presente nos diabéticos, parece estar mais realcionada a neuropatia diabética do que a deficiência da insulina. $O$ volume diastólico (VD) do ventrículo esquerdo não aumenta com o exercício, diferente do que ocorre em indivíduos saudáveis. $\mathrm{A} \mathrm{VO}_{2}$ máxima é reduzida, bem como a capacidade aeróbica, chegando a $20 \%$ de perda, mesmo com reposição de insulina. ${ }^{11}$

No entano, existem muitas controvérsias em relação às alterações metabólicas com bom controle glicêmico. ${ }^{7}$ Bally, Laimer, Stettler, ${ }^{6}$ por meio de artigo de revisão, identificaram não haver grandes diferenças na resposta da glicose relacionada ao exercício em pessoas saudáveis ou com DM1 em uso adequado de insulina, pois o armazenamento de glicogênio no músculo es- 
quelético e no fígado ocorrem de maneira semelhantes nos dois grupos. ${ }^{6}$ Outro artigo de revisão, concluiu que a capacidade aeróbica apresenta resultados bastante parecidos na ausência de neuropatia periférica. ${ }^{11}$ Estudo de caso controle avaliou a taxa de oxidação da glicose em pacientes com DM1 em condições normais de vida em exercícios prolongados ( $3 \mathrm{hs}$ ), identificando que a concentração de insulina e glicemia foram significativamente maiores em pacientes durante todo o exercício. A oxidação da glicose diminuiu significativamente com o aumento da duração do exercício, porém sem diferenças significativas nos dois grupos. ${ }^{7}$

Estudo descritivo transversal, realizado com adolescentes diabéticos, identificou a redução de $21 \%$ na glicemia pós exercício quando utilizada a técnica de atividade aeróbica com sprints. ${ }^{12} \mathrm{O}$ sprint consiste em intercalar exercícios de baixa e alta intensidade. Esta mesma técnica, aplicada em pacientes adultos, demonstrou menor risco de hipoglicemia pós exercício, aumento da secreção do hormônio do crescimento, catecolameninas e lactato quando utilizadas atividades físicas intervaladas ao invés de atividades continuadas. ${ }^{13}$

O exercício físico altera as vias do metabolismo melhorando o controle, porém vários fatores precisam ser avaliados durante todo o processo a fim de evitar a hiperglicemia que pode ocorrer após exercícios intensos e vigorosos ou hipoglicemia, que é mais comum em exercícios mais longos e moderados. ${ }^{11}$ Sprints apresentam efeito estabilizador da glicose, porém necessita de adaptação de insulina para previnir hipoglicemias. Exercícios intensos de apenas 10 segundos podem provocar mudança na sensibilidade da insulina periférica. Comparando o resultado de exercícios intermitentes de alta intensidade e exercícios moderados contínuos, por ensaio clínico prospectivo randomizado e aberto, foram identificados níveis semelhantes de produção de glicose hapática, porém a eliminação da glicose foi significativamente menor no grupo que realizou sprints. ${ }^{13}$

Nos adolescentes existe uma estreita relação entre os níveis eleveados de hemoglobina glicosilada ( $\mathrm{HbA} 1 \mathrm{c}$ ) e sobrepeso ou obesidade. ${ }^{12}$ Porém, não há comprovação de que o exercício físico melhore os níveis de hemoglobina glicosildada, mas sabe-se que em indivíduos ativos a morbimortalidade diminui independente do resultado da $\mathrm{HbA} 1 \mathrm{c}$. Alterações na resposta dos exercícios não deveriam desencorajar as atividades físicas, mas estimular a busca pela identificação dos exercícios que maximizem efeitos benéficos para a saúde. ${ }^{11}$

\section{EXERCÍCIO FÍSICO NA DIABETES TIPO 2}

Exercícios físicos têm sido utilizados como tratamentos não farmacológico no DM devido ao seu efeito hipoglicemiante, porém nos indivíduos com alterações do metabolismo de carboidratos a redução glicêmica não é tão evidente. ${ }^{14}$ O DM2 altera o metabolismo de glicose e aminoácidos não somente durante o exercício, mas também durante o período de recuperação. ${ }^{9}$

Efeito agudo do exercício físico aeróbico sobre a glicemia capilar em três grupos foi avaliado por estudo prospectivo quase experimental. O primeiro grupo, formado por sete indivíduos diabéticos que utilizavam antidiabéticos orais; o segundo, oito indivíduos não diabéticos; e o terceiro, oito indivíduos diabéticos que faziam uso de insulina. Os três grupos foram submetidos ao protocolo de exercícios de 70 minutos, incluindo aquecimento, caminhada, alongamento e relaxamento. Neste estudo, a ação hipoglicemiante foi evidenciada nos indivíduos não diabéticos. ${ }^{15}$

Estudo de caso controle avaliou 139 metabólitos e hormônios plasmáticos em três tempos, durante exercício de bicilceta de 60 minutos e uma fase de recuperação de 180 minutos em pacientes com DMT2 e controles saudáveis classificados por idade, porcentagem de gordura corporal e consumo máximo de oxigênio $\left(\mathrm{VO}_{2}\right)$. O resultado apontou para melhora no metabolismo de glicose e aminoácidos em pacientes diabéticos quando comparados aos controles. ${ }^{9}$

Efeitos agudos do exercício também foram avaliados por crossover considerando dois grupos de intervenção e um grupo controle. O primeiro grupo de intervenção realizou ciclos repetidos de 3 minutos alternando entre caminhada lenta e rápida durante uma hora. O segundo grupo realizou caminhada contínua pelo mesmo período. O consumo de oxigênio $\left(\mathrm{VO}_{2}\right)$ e a glicose foram monitorados continuamente. O grupo de atividade intercalada apresentou menor glicemia plasmática máxima e maior taxa de depuração metabólica de glicose..$^{15}$

Nos pacientes com DM2 também se observa que o treinamento intervalado de alta intensidade melhora o controle glicêmico e a função pancreática. Estudo de caso controle acompanhou por 8 semanas o treinamento intervalado de alta intensidade e baixo volume em pacientes com DM2 e controles saudáveis. Os resultados demonstram que ambos os grupos atingiram 90\% da frequência cardíaca máxima e apresentaram perdas significativas da massa abdominal e circunferência da cintura, sendo que a perda de gordura parece depender da intensidade. Nos pacientes com DM2 houve controle glicêmico e diminuição da tolerância à glicose e hemoglobina glicada. As concetrações de glicose no plasma em jejum e pós-prandial permaneceram inalteradas. Este estudo apresentou um resultado inusitado: o consumo máximo de oxigênio pulmonar absoluto e relativo foi significativamente aumentado nos indivíduos que praticaram sprints. ${ }^{10}$

Medir continuamente a glicose a fim de identificar quantas refeições um único exercício poderia reduzir as respostas de glicemia pós-prandial nas refeições subsequantes em pacientes com DM2 que realizou exercício e o grupo controle que não realizou nenhuma atividade física. O grupo que realizou atividade física reduziu significativamente a glicose nas primeiras 24 hs e diminuiu a glicose pós-prandial para todas as refeições durante dois dias. Exercícios diários podem ser necessários para o controle da glicemia pós-prandial. ${ }^{14}$

Inúmeros artigos tratam do tema da atividade física, porém esta revisão narrativa encontrou limitações, pois os artigos selecionados representavam a complexidade e diversidade do tema em relação à DM1 e DM2, mostrando inclusive, resultados inusitados e contraditórios.

\section{CONSIDERACÕES FINAIS}

Não foi possível concluir qual o tipo de exercício físico, volume e intensidade é ideal para pacientes com DM1 e DM2. Para aproveitar ao máximo todos 
os benefícios dos exercícios físicos é relevante que os mesmos sejam estruturados de forma individualizada, considerando a alimentação, a administração de insulina exógena conforme a duração e a intensidade da atividade física. Desta forma, será possível obter resultados positivos em relação à capacidade cardiorespiratória e a regulação da glicose e da insulina. Estudos mais aprofundados, que avaliem os diversos tipos de exercício físico, volume e intensidade, são necessários.

A adesão, motivação e tempo para seguir as recomendações referente aos exercícios físicos ainda representam grandes desafios. Desta forma, há necessidades crescentes de políticas públicas de saúde com foco no manejo interdisciplinar para tratamento e prevenção do DM, que incluam o exercício físico ideal para cada indivíduo no seu projeto terapêutico.

\section{REFERÊNCIAS}

1. Milech A, Oliveira JEP, Vencio S. Diretrizes da Sociedade Brasileira de Diabetes (2015-2016). São Paulo: A. C. Farmacêutica, 2016.

2. WHO. World Health Organization. Global Report on Diabetes. 2016. Disponível em: <http://apps.who.int/ iris/bitstream/10665/204871/1/9789241565257_eng. pdf?ua=1>. Acesso em: 07 set. 2017.

3. IDF. International Diabetes Federation. IDF Diabetes Atlas. $6^{\mathrm{a}}$ ed. Brussels: International Diabetes Federation, 2014 Disponível em: <http://www.idf.org/diabetesatlas>. Acesso em: 07 set. 2017.

4. Brasil, Ministério da Saúde. Secretaria de Atenção à Saúde. Departamento de Atenção Básica. Estratégias para o cuidado de pessoas com doença crônica: diabetes mellitus. $1^{\text {a }}$ ed. Brasília: Ministério da Saúde, 2013.

5. Roque FR, Hernanz R, Salaices M, Briones A. Exercise training and cardiometabolic disease: focus on the vascular sistem. Curr Hypertens Res 2013;15(3). doi: 10.1007/ s11906-013-0336-5

6. Bally L; Laimer M; Stettler C. Exercise-associated glucose metabolism in individuals with type 1 diabetes mellitus. Current Opinion in Clinical Nutrition \& Metabolic Care 2015;18(4):428433. doi: 10.1097/MC0.0000000000000185

7. Geat M, Stel G, Poser S, Driussi C, Stenner E, Francescato MP. Whole-body glucose oxidation rate during prolonged exercise in type 1 diabetic patients under usual life conditions. Metabolism 2013;62(6):836-44. doi: 10.1016/j. metabol.2013.01.004

8. Barrile SR, Caneglian CB, Gimenes C, Conti MHS, Arca EA, Rosa Junior G, Martinelli B. Efeito agudo do exercício aeróbico na glicemia em diabéticos 2 sob medicação. Rev Bras Med Esporte 2015;21(5):360-363. doi: 10.1590/1517869220152105117818

9. Hansen JS, Zhao X, Irmler M, Liu X, Hoene M, Scheler M, Li $Y$, Beckers J, Hrabl de Angelis M, Häring HU, Pedersen BK, Lehmann R, Xu G, Plomgaard P, Weigert C. Type 2 diabetes alters metabolic and transcriptional signatures of glucose and amino acid metabolis during exercise and recovery. Diabetologia 2015;58(8):1845-54. doi: 10.1007/s00125015-3584-x

10. Madsen SM, Thorup AC, Overgaard K, Jeppesen PB. High intensity interval training improves glycaemic control and pancreatic $B$ cell function of type 2 diabetes patients. PLoS One 2015;10(8)e0133286. doi: 10.1371/journal. pone.0133286

11. Galassetti $P$, Riddell MC. Exercise and type 1 diabetes (T1DM). Compr Physiol 2013;3(3):1309-36. doi: 10.1002/ cphy.c110040

12. Lima VA, Mascarenhas LPG, Decimo JP, Souza WC, França $\mathrm{SN}$, Leite N. Efeito agudo dos exercícios intermitentes sobre a glicemia de adolescentes com diabetes tipo 1. Rev Bras Med Esporte 2017;23(1):12-15. doi: 10.1590/1517869220172301158088

13. Bally L, Zueger T, Buehler T, Dokumaci AS, Speck C, Pasi N, Carlos C. Paganini D, Feller K, Loher H, Rosset R, Wilhelm M, Tappy L, Boesch C, Stettler C. Metabolic and hormonal response to intermittent high-intensity and continuous moderate intensity exercise in individuals with type 1 diabetes: a randomised crossover study. Diabetologia 2016;59:776-784. doi: 10.1007/s00125-015-3854-7

14. Barrile SR, Coneglian CB, Gimenes C, Conti MHS, Arca EA, Rosa Junior G, Martinelli B. Efeito agudo do exercício aeróbico na glicemia em diabéticos 2 sob medicação. Rev Bras Med Esporte 2015;21(5):360-363. doi: 10.1590/1517869220152105117818

15. Karstoft K, Christensen CS, Pedersen BK, Solomon TP. The acute effects of interval - Vs continuous-walking exercise on glycemic control in subjects with type 2 diabetes: a crossover, controlled study 2014;99(9):3334-42. doi: $10.1210 /$ jc. $2014-1837$

Como citar: ELY, Karine Zenatti et al. Exercício físico na diabetes mellitus, uma revisão narrativa. Cinergis, Santa Cruz do Sul, v. 18, dez. 2017. ISSN 2177-4005. Disponível em: <https://online.unisc.br/seer/index.php/cinergis/article/view/11175>. Acesso em: 16 jan. 2018. doi:http://dx.doi.org/10.17058/cinergis.v18i0.11175. 